\title{
La libre détermination des personnes et le contrôle de l'Etat dans la bioéthique aujourd'hui
}

Jean Martin

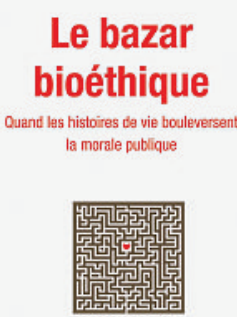

Véronique Fournier Le bazar bioéthique

Paris: Laffont; 2010 211 pages, $36.50 \mathrm{CHF}$. ISBN 978-2-221-11505-3

jean.martin@saez.ch
Dans le domaine en évolution rapide de la bioéthique, la place et l'importance du pouvoir normatif de l'Etat, fixant par la loi des interdictions, des contraintes, des droits de regard, sont débattues et parfois contestées. Comme est discutée la mesure dans laquelle il existe ou pas - des principes absolus, de nature ontologique notamment, représentant des impératifs dont la société occidentale de tradition judéo-chrétienne - voire la communauté universelle - ne saurait s'éloigner. Récemment, le BMS a publié des prises de position qui ont donné lieu à de vifs échanges $[1,2]$ et plusieurs lettres de lecteur y relatives.

\section{«Le bazar bioéthique»}

C'est le titre de l'ouvrage de Véronique Fournier [3], cardiologue et médecin de santé publique, qui a été chargée dans le cabinet du Ministre Bernard Kouchner de la préparation de la loi sur les droits de la personne malade de 2002, et a créé à cette époque le Centre d'éthique clinique de l'hôpital Cochin, à Paris, qu'elle dirige. Le livre est le fruit des enseignements rassemblés au cours des entretiens, évaluations et recherches réalisés dans ce centre; ses chapitres traitent successivement des donneurs vivants d'organes (foie surtout), de la recherche de l'enfant parfait, y compris au moyen du diagnostic pré-implantatoire, de transsexualisme et des opérations de changement de sexe/ genre, des situations-limites en matière de procréation médicalement assistée (PMA).

Dit ici en toute modestie, je me suis procuré ce livre par acquit de conscience, parce qu'il ne faut pas s'intéresser seulement à ce qui est publié en anglais; en m'attendant à un exposé à l'appui des règles et spécificités françaises dans le domaine. Et c'est avec une surprise bienvenue que, dès les premières pages, on découvre une pensée indépendante, différenciée, basée sur une pratique propre - à la différence d'«experts de salon» parfois éloignés de ce que vivent, dans la communauté et son système de santé, patient(e)s et professionnels.

Sur la base de son expérience et de celle de son centre (une grande partie du livre decrit des situations vécues), Fournier apporte entre autres des éclairages substantiels sur les dimensions collectives des enjeux bioéthiques, et sur la légitimité ou pas - aujourd'hui et dans l'avenir - de l'intervention de la forte main de $l^{\prime}$ Etat, et dans quelle mesure. Elle discute notamment le lien, actuellement indiscuté dans la conception française, entre le devoir des pouvoirs publics de mettre à disposition des soins de qualité, tenant compte de l'évolution des techniques - du progrès de la médecine - et le devoir de financer lesdits soins. Elle pose la question de savoir si on ne sera pas amené, dans l'un ou l'autre des domaines traités, à séparer les deux choses: à savoir envisager que des prestations puissent être rendues disponibles mais sans que l'Etat en assume la charge financière (avec, dans un tel modèle, les interrogations que suscite la survenue d'inégalité sociales dans l'accès à certaines possibilités).

\section{La question du consentement éclairé des personnes et de sa vérification par des experts} Problématique très actuelle. Quand et jusqu'où convient-il que les pouvoirs publics prévoient le contrôle du bon sens des demandeurs de soins (patients, donneurs d'organes, couples s'adressant à la PMA), du caractère raisonnable de leur projets de vie, ou parentaux, ou de changement de sexe/genre. Des règles sont nécessaires mais on voit aussi des propositions de réglementation qui donnent l'impression que, forcément, l'Etat sait mieux que des adultes capables de discernement ce qui est bon pour eux... Au sujet d'un projet de loi censé lever l'interdiction en Suisse du diagnostic préimplantatoire, nous disions «Le lecteur est frappé par une orientation paternaliste: un contrôle serré et pour chaque cas de l'Etat (sage) serait indispensable pour éviter les dérives des couples (irresponsables?) désireux de concevoir» [4]. Une crainte similaire vaut pour un projet de réglementation restrictive - proposé puis retiré - de l'assistance au suicide, qui tendait à déresponsabiliser les acteurs essentiels dans ce cas, à savoir un malade et le médecin dont il sollicite une prescription létale [5].

A propos de la qualité de la démarche et du consentement de possibles donneurs vivants d'organes, Véronique Fournier écrit «il est tout à fait insuffisant, voire parfaitement inadéquat, d'aborder [les situations à examiner] sur un plan [au moyen de critères] purement théorique, à distance de ce qui se vit pour les personnes concernées.» Et elle souligne avoir appris que le raisonnement qui voulait confier à un centre d'éthique comme le sien l'appréciation du donneur uniquement (et pas celle du receveur) était insatisfaisante.

Plus avant sur cette question centrale «Même si les circonstances familiales étaient telles que l'on pouvait se poser des questions (...), quel contexte familial en 
est-il exempt et en quoi était-ce notre affaire? N'étaitce pas nous immiscer un peu loin dans la vie des gens? (...) L'expérience m'a conduite à consolider ma méfiance. Il ne me paraît pas opportun d'émettre, ni moi ni du reste quiconque, un avis sur le consentement du donneur (...). Au fil de l'expérience, la qualité du consentement est devenue pour moi une mauvaise question.» De plus, concrètement dit-elle, «La procédure est maintenant devenue si lourde qu'elle en devient presque suspecte» (question qu'on peut poser en Suisse en ce qui concerne la réglementation de la médecine de transplantation).

\section{La juste mesure - ou démesure - du contrôle exercé par les pouvoirs publics sur des actes médicaux}

Il n'est pas bon que l'Etat se mêle de tout, en particulier de situations personnelles/familiales intimes (un Etat bon à tout est un Etat bon à rien, disait un des magistrats avec qui nous avons travaillé). Dans le chapitre consacré au transsexualisme, Véronique Fournier discute/soupèse les poids respectifs que doivent avoir les souffrances et demandes des personnes concernées, d'une part, et les limites que poserait l'Etat d'autre part. En arrivant à une conclusion qui peut surprendre: «Du reste, peut-être vaut-il mieux que le transsexualisme ne soit pas reconnu comme une question de bioéthique puisque la sanction législative en serait alors probablement restrictive, comme c'est le cas pour tout ce qui relève de la loi de bioéthique jusqu'à présent, tendant à privilégier l'intérêt de la société sur celui de l'individu.»

A propos de PMA et des évaluations y relatives: «La conception même d'un garde-fou mis en place par la société pour protéger les enfants des excès de leur parents leur était incompréhensible [aux couples demandeurs]. Qui mieux qu'eux connaissait le vrai prix à payer de tel ou tel choix? En revanche, ils comprenaient que leur médecin ait besoin de réfléchir avec d'autres afin de les guider au mieux.» Fournier cite l'avocat parisien Pierre Verdier, spécialiste du domaine, disant qu'il n'avait jamais vu de parents faire passer leur prétendu intérêt avant celui de l'enfant...

La loi jusqu'ici a été conçue comme défensive, contre la «folie des hommes». Fournier plaide pour que la législation évolue dans le sens général des droits des malades, de la solidarité vis-à-vis d'eux, plutôt que d'être «loi d'interdiction morale au service de la préservation d'une quelconque bonne conscience ou bien-pensance collective».

Observant une certaine institutionnalisation de l'éthique, l'auteure exprime son «sentiment d'injustice à voir la façon dont la société traite aujourd'hui [les demandes présentées], en acceptant certaines, en condamnant les autres, sur des critères supposés clairs, bâtis autour d'une ligne de démarcation qui est officiellement la maladie, mais dont l'appréciation est éminemment subjective et beaucoup plus morale qu'autre chose.»

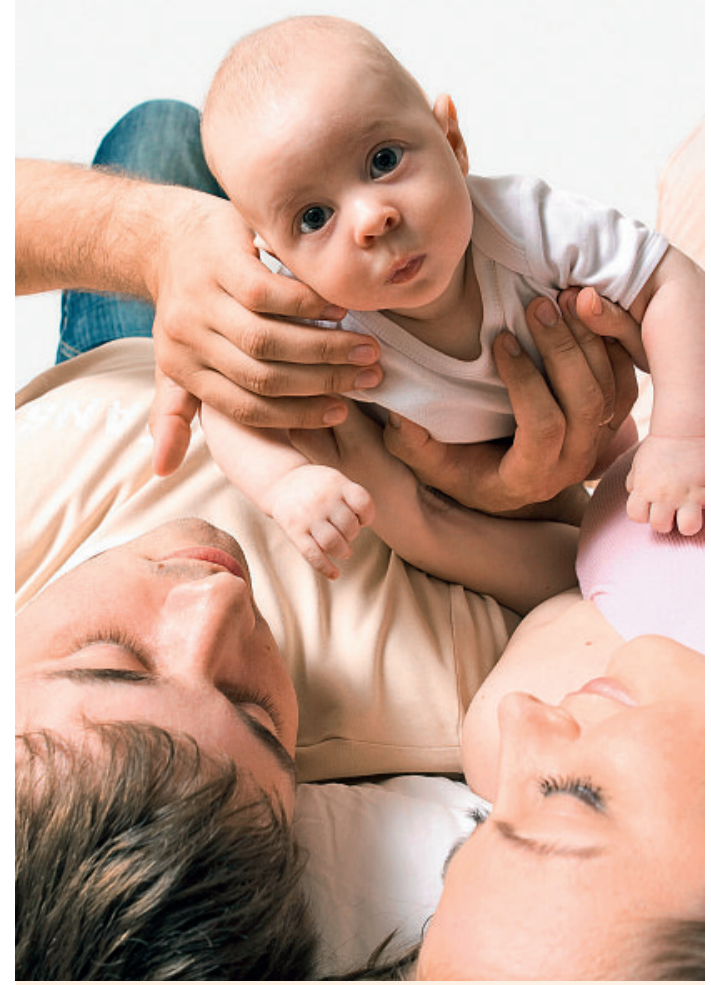

Un chapitre du livre de Véronique Fournier traite la recherche de l'enfant parfait, par ex. par diagnostic pré-implantatoire.

On doit tenir compte de la transformation sociale. Parmi les valeurs sur lesquelles il convient de s'appuyer, le souci de l'autre est au premier rang. Pour les personnes concernées (donneurs vivants d'organes, parents demandant un retrait thérapeutique s'agissant de leur enfant nouveau-né gravement handicapé, demandeurs de PMA) «il leur a fallu puiser à la source du meilleur d'eux-mêmes, à l'endroit où l'autre est plus important que soi. Ils le font avec un courage qui force le respect».

\section{Du rôle de la médecine et des médecins}

Véronique Fournier se préoccupe de ce qu'est le mandat de la médecine et des médecins dans la société. Sujet majeur. Elle déplore qu'on n'accorde pas aujourd'hui l'attention voulue à l'indépendance nécessaire de la profession. Les médecins «se sentent délégitimés par les demandeurs comme par la société. Pour autant, ils répugnent à se voir réduits à jouer les prestataires de services, sommés d'obtempérer sans discussion (...) risque de dériver petit à petit vers une médecine agissant sur ordre, ayant perdu ses repères de bonne pratique.» A propos de la volonté d'un couple rencontré de recourir à la PMA avec le risque de naissance d'un deuxième enfant handicapé: A leurs yeux, «qui mieux que les parents connaissait ce qu'était la vie avec ce handicap? (...) Je me suis surprise dans ce cas à plutôt pencher du côté [de la réticence] de mes confrères. Etaient-ils là pour être au 
simple service de la demande de ces parents, y compris au risque de participer à créer de la souffrance quand leur métier était de faire l'inverse? Comme des prestataires en quelque sorte, n'ayant pas leur mot à dire et tenus de fournir la technique dont ils sont experts.» A l'évidence, il y a va de notre intégrité et de notre déontologie professionnelles. A plusieurs égards, il convient aujourd'hui d'examiner de manière critique les poussées vers plus de textes législatifs ou réglementaires, et de revaloriser plutôt l'éthique et la responsabilité propres du médecin [6].

«Il m'apparaît de plus en plus clairement que la médecine doit se considérer comme un authentique troisième acteur indépendant entre le patient et la société.»

\section{La question de fond}

Dans une société en évolution rapide, qu'est-ce qui doit rester immuable et qu'est-ce qui peut/doit évoluer? (cf. début de ce texte). Il faut, dit Fournier, «discuter la question de fond: le corpus éthique d'une société doit-il être celui qui traduit la façon dont les gens vivent et font leurs choix au quotidien ou doit-il rester un corpus idéal, pensé par des sages choisis pour savoir nous guider afin que nous ne perdions pas notre âme?».

«Ce sont des évolutions socioculturelles qui sont à l'œuvre dans le débat actuel autour des questions de fin de vie. La reviviscence récente des interrogations s'explique tout autant par un certain changement dans la façon de penser la sacralité de la vie et de la mort [que par les avancées de la médecine].»

Un exemple: de plus en plus souvent, on va voir des situations où des personnes iront chercher et obtenir ailleurs une aide - en matière de PMA - par des modalités interdites en Suisse: don d'ovocyte ou d'embryon, mère porteuse, couples homosexuels ayant (fait faire) un enfant. Ces personnes seront menacées de poursuites pénales et/ou rencontreront des difficultés graves en termes de droit civil/état civil. La question est alors: aussi longtemps que de tels cas restent exceptionnels, la société vivra tant bien que mal avec la dissonance qu'ils créent. Mais que fait-on, que recommande par exemple une Commission nationale d'éthique, quand ils se multiplient comme ils le feront très vraisemblablement dans l'avenir? Malgré les infractions qui ont permis à ces enfants de naître (!), on devra impérativement d'un point de vue d'éthique sociale leur garantir les mêmes droits et possibilités qu'à leurs contemporains; si on ne le faisait pas, ils n'auraient nulle part où se tourner - et on n'imagine guère que, après avoir surmonté de nombreux ob- stacles, un couple homosexuel dans ce cas donne son enfant pour adoption... A l'évidence, nos systèmes civils et politiques seront mis au défi de trouver des solutions acceptables.

«Dissolution progressive du consensus social (...). Les limites de la maladie sont devenues, comme celles de la morale, trop sujettes à caution pour servir de référence». Well... fort de café, mais il y a de cela dans la situation dans laquelle nous sommes.

\section{Pistes pour I'avenir}

Un des constats de V. Fournier quinze ans après la promulgation en 1994 des lois françaises de bioéthique et les débats autour d'elles: «on peut se demander si les dangers dont on nous menaçait - la catastrophe annoncée - ne tenaient pas davantage du fantasme que d'une quelconque réalité (...). Sur le terrain, c'est plutôt une conscience aiguë de leur responsabilité qu'expriment les couples en désir d'enfant, ils sont loin du consumérisme mâtiné de déliquescence morale dont on les soupçonne».

\section{Parmi les valeurs sur lesquelles \\ il convient de s'appuyer, le souci de l'autre est au premier rang}

De son expérience comme responsable d'un Centre d'éthique clinique, le premier enseignement que Fournier tire, "peut-être le plus important, consiste à faire le pari de l'autre en tant que personne; se laisser conduire par ce que demandent les premiers concernés, qu'il s'agisse de personnes malades ou d'autres; leur faire confiance plutôt que de s'en méfier (...) Je voudrais faire partager ma conviction que les gens sont tous des agents éthiques, des personnes dont le cœur, le noyau existentiel, est éthique.»

Dernières lignes du livre: elle appelle de ses vœux un avenir de la bioéthique marqué par «L'individu plutôt que le collectif, le juste plutôt que le bien, la solidarité plutôt que l'égalité, la lutte sans merci contre l'exploitation de l'homme du Sud par l'homme du Nord [faisant référence aux femmes de pays/milieux défavorisés donneuses d'ovocytes ou mères porteuses], la refondation de la médecine dans la relation médecin-malade: il faut bien que les idéaux soient ambitieux, si l'on veut qu'ils soient porteurs d'avenir».

\footnotetext{
Höffe O. Philosophische
Ethik: Fahne im Wind oder
Fels in der Brandung?
Bull Méd Suisses. 2010;
91(32):1199-1202.
Fischer J. Ethik als Prinzipien
ethik? Bull Méd Suisses. 2010
91(38):1484-7.
Fournier V. Le bazar
bioéthique - Quand les
histoires de vie bouleversent
la morale publique. Paris:
Robert Laffont; 2010.
Martin J. DPI - Un projet
loi incompréhensible. Bull
Méd Suisses. 2009;90(13):552
(Publié en allemand sous
le titre «PID - ein unverstän
licher Gesetzesentwurf»).
Martin J. Assistance au
suicide: surveiller tout en
donnant sa place à l'éthique.
Bull Méd Suisses. 2010;91(7);
286. (Publié en allemand
sous le titre «Suizidbeihilfe
beaufsichtigen und ethisch
handeln»).
Martin J. De l'éthique au
droit... et retour? Bull Méd
Suisses. 2010;91(17):698.
(Publié en allemand sous
le titre («Von der Ethik zum
Recht ... und wieder

Recht ... und wieder zurück?»).

Höffe O. Philosophische
Ethik: Fahne im Wind ode
Fels in der Brandung?
Bull Méd Suisses. 2010;
91(32):1199-1202.
Fischer J. Ethik als Prinzipien
ethik? Bull Méd Suisses. 2010
91(38):1484-7.
Fournier V. Le bazar
bioéthique - Quand les
histoires de vie bouleverse
la morale publique. Paris:
Robert Laffont; 2010.
Martin J. DPI - Un projet
loi incompréhensible. Bull
Méd Suisses. 2009;90(13):552
(Publié en allemand sous
le titre «PID - ein unverständ
licher Gesetzesentwurf»).
Martin J. Assistance au
suicide: surveiller tout en
donnant sa place à l'éthiq
Bull Méd Suisses. 2010;91(7);
286. (Publié en allemand
sous le titre «Suizidbeihilfe:
beaufsichtigen und ethisch
handeln»).
Martin J. De l'éthique au
droit... et retour? Bull Méd
Suisses. 2010;91(17):698.
(Publié en allemand sous
le titre («Von der Ethik zum
Recht ... und wieder

Höffe O. Philosophische
Ethik: Fahne im Wind ode
Fels in der Brandung?
Bull Méd Suisses. 2010;
91(32):1199-1202.
Fischer J. Ethik als Prinzipien
ethik? Bull Méd Suisses. 2010
91(38):1484-7.
Fournier V. Le bazar
bioéthique - Quand les
histoires de vie bouleversent
la morale publique. Paris:
Robert Laffont; 2010.
Martin J. DPI - Un projet de
loi incompréhensible. Bull
Méd Suisses. 2009;90(13):5
(Publié en allemand sous
le titre «PID - ein unverständ
licher Gesetzesentwurf»).
Martin J. Assistance au
suicide: surveiller tout en
donnant sa place à l'éthique
Bull Méd Suisses. 2010;91(7):
286. (Publié en allemand
sous le titre «Suizidbeihilfe
beaufsichtigen und ethisch
handeln»).
Martin J. De l'éthique au
droit... et retour? Bull Méd
Suisses. 2010;91(17):698.
(Publié en allemand sous
le titre («Von der Ethik zun
Recht .. und wieder
and

Höffe O. Philosophische
Ethik: Fahne im Wind oder
Fels in der Brandung?
Bull Méd Suisses. 2010;
91(32):1199-1202.
Fischer J. Ethik als Prinzipien
ethik? Bull Méd Suisses. 2010
91(38):1484-7.
Fournier V. Le bazar
bioéthique - Quand les
histoires de vie bouleversent
la morale publique. Paris:
Robert Laffont; 2010.
Martin J. DPI - Un projet
loi incompréhensible. Bull
Méd Suisses. 2009;90(13):552
(Publié en allemand sous
le titre «PID - ein unverstän
licher Gesetzesentwurf»).
Martin J. Assistance au
suicide: surveiller tout en
donnant sa place à l'éthique.
Bull Méd Suisses. 2010;91(7);
286. (Publié en allemand
sous le titre «Suizidbeihilfe
beaufsichtigen und ethisch
handeln»).
Martin J. De l'éthique au
droit... et retour? Bull Méd
Suisses. 2010;91(17):698.
(Publié en allemand sous
le titre («Von der Ethik zum
Recht ... und wieder

Höffe O. Philosophische
Ethik: Fahne im Wind oder
Fels in der Brandung?
Bull Méd Suisses. 2010;
91(32):1199-1202.
Fischer J. Ethik als Prinzipien
ethik? Bull Méd Suisses. 2010
91(38):1484-7.
Fournier V. Le bazar
bioéthique - Quand les
histoires de vie bouleversent
la morale publique. Paris:
Robert Laffont; 2010.
Martin J. DPI - Un projet
loi incompréhensible. Bull
Méd Suisses. 2009;90(13):552
(Publié en allemand sous
le titre «PID - ein unverstän
licher Gesetzesentwurf»).
Martin J. Assistance au
suicide: surveiller tout en
donnant sa place à l'éthique.
Bull Méd Suisses. 2010;91(7);
286. (Publié en allemand
sous le titre «Suizidbeihilfe
beaufsichtigen und ethisch
handeln»).
Martin J. De l'éthique au
droit... et retour? Bull Méd
Suisses. 2010;91(17):698.
(Publié en allemand sous
le titre («Von der Ethik zum
Recht ... und wieder
}

(n) 